\title{
Incidence of small bowel neoplasia in Lynch syndrome assessed by video capsule endoscopy
}

\section{(ㄷ)(1) $\odot$}

\section{Authors}

Jasmijn F. Haanstra' ${ }^{1,7}$, Abdul Al-Toma², Evelien Dekker³, Steven A.L.W Vanhoutvin ${ }^{4}$, Fokko M. Nagengast ${ }^{5}$, Elisabeth M. Mathus-Vliegen ${ }^{3}$, Monique E. van Leerdam ${ }^{4,6}$, Wouter H. de Vos tot Nederveen Cappel ${ }^{7}$, Roeland A. Veenendaal ${ }^{8}$, Annemieke Cats ${ }^{4}$, Silvia Sanduleanu ${ }^{9}$, Hans F.A. Vasen ${ }^{8,10}$, Jan H. Kleibeuker ${ }^{1}$, Jan J. Koornstra ${ }^{1}$

Institutions

1 Department of gastroenterology and hepatology, University of Groningen, University Medical Center Groningen, Groningen, The Netherlands

2 Department of gastroenterology and hepatology, St Antonius Hospital, Nieuwegein, The Netherlands

3 Department of gastroenterology and hepatology, Amsterdam Medical Center, Amsterdam, The Netherlands

4 Department of gastroenterology and hepatology, The Netherlands Cancer Institute-Antoni van Leeuwenhoek Hospital (NKI-AVL), Amsterdam, The Netherlands

5 Department of gastroenterology and hepatology, Radboud University Nijmegen Medical Center, Nijmegen, The Netherlands

6 Dept of gastroenterology and hepatology, Erasmus Medical Center, Rotterdam, The Netherlands

7 Department of gastroenterology and hepatology, Isala, Zwolle, The Netherlands

8 Department of gastroenterology and hepatology, Leiden University Medical Center, Leiden, The Netherlands

9 Department of gastroenterology and hepatology, Maastricht University Medical Center, Maastricht, The Netherlands

10 The Netherlands Foundation for the Detection of Hereditary Tumours, Leiden, The Netherlands

submitted 15.12.2016

accepted after revision 15.5.2017

\section{Bibliography}

DOI https://doi.org/10.1055/s-0043-111723 |

Endoscopy International Open 2017; 05: E622-E626

(c) Georg Thieme Verlag KG Stuttgart · New York

ISSN 2364-3722

\section{Corresponding author}

J. J. Koornstra, MD, PhD, Department of Gastroenterology and Hepatology, University Medical Center Groningen, P.O. Box 30001, 9700 RB Groningen, the Netherlands Fax: +310503619306

j.j.koornstra@umcg.nl

\section{ABSTRACT}

Background and study aims Lynch syndrome (LS) patients have an increased risk of small bowel cancer. The question is whether surveillance will lead to early detection of (pre)malignant lesions. We recently reported on prevalence of small bowel neoplasia (SBN) in LS patients as assessed by video capsule endoscopy (VCE). The aim of this prospective study was to determine the incidence of SBN.

Patients and methods Asymptomatic LS patients who underwent a VCE were invited to undergo a second VCE procedure 2 years later. If abnormalities or polypoid lesions larger than $1 \mathrm{~cm}$ were detected, subsequent endoscopic procedures were performed.

Results A total of 155 (78\%) of the initial 200 patients underwent a second VCE procedure after a mean of 2.2 (range $1-6)$ years. In 17 of the 155 (11\%) patients possibly significant lesions were detected, which required further investigation by means of gastroduodenoscopy $(n=8)$ or balloonassisted endoscopy $(n=9)$. These procedures revealed no SBN.

Conclusion No SBN was found after 2 years. Surveillance of the small bowel by VCE does not seem to be warranted in asymptomatic LS patients.

This study was registered in the Clinical Trials.gov registry with identifier NCT00898768.

\section{Introduction}

The most common inherited condition associated with colorectal cancer is Lynch syndrome (LS), formerly known as hereditary non-polyposis colorectal cancer (HNPCC). The syndrome is caused by a defect in one of the mismatch repair (MMR) genes (MLH1, MSH2/Epcam, MSH6, or PMS2) [1]. Besides a high lifetime colorectal cancer risk, MMR-gene mutation carriers also 
have an elevated risk of developing various extracolonic malignancies, including small bowel cancer [2].

Compared with the general population, the relative risk of developing small bowel carcinoma is more than 100 in LS patients, with an estimated lifetime risk of $4.2 \%[3,4]$. Like LSassociated colorectal cancer, it also occurs at a relatively early age. Retrospective analyses showed a median age at diagnosis of 39 (range $11-81$ ) years [3,5]. Recently, we showed that the prevalence of small bowel neoplasia (SBN) in asymptomatic LS patients as assessed by video capsule endoscopy (VCE) was markedly lower than previously estimated $[6,7]$. SBN was present in only 3 of the 200 patients (1.5\%) who underwent a VCE procedure ( 2 duodenal adenocarcinomas and 1 duodenal tubulovillous adenoma with low grade dysplasia) [6].

LS patients benefit from colonic surveillance $[8,9]$. In order to determine whether they might also benefit from surveillance of the small bowel more data are needed. We therefore performed a follow-up study in the original study cohort to assess newly developed (incidence) or possibly missed SBN in LS patients by VCE.

\section{Patients and methods}

\section{Study design}

As reported earlier [6], this study was a nationwide prospective multicenter trial in the Netherlands that aimed to assess the prevalence and incidence of SBN in asymptomatic LS patients by VCE. The study was registered in the ClinicalTrials.gov registry (NCT00898768). The study protocol was approved by the local Medical Ethics Committee of the University Medical Center Groningen and by all participating centers.

\section{Study procedures and population}

All 200 previously included asymptomatic proven carriers of a MMR-gene mutation (MLH1, MSH2/Epcam, MSH6, or PMS2) aged between 35 and 70 years at the date of inclusion were invited for a second VCE procedure 2 years after the first procedure, unless in the meantime exclusion criteria were met. Because exact data on the prevalence and incidence of small bowel neoplasms are scarce, the interval of 2 years was chosen based on the recommendations made for colonoscopic surveillance.

Exclusion criteria were: (1) previous small bowel surgery or large bowel surgery involving the ileocecal valve; (2) a strong clinical suspicion of small bowel stricture; (3) pregnancy; and (4) presence of any psychological, familial, sociological, or geographical condition potentially hampering compliance with the study protocol and follow-up schedule.

A standardized regimen of $2 \mathrm{~L}$ bowel preparation based on polyethylene glycol (PEG) electrolyte solution was given, starting the day before the procedure. The video capsules used were supplied by Given Imaging (Yoqneam, Israel), with a recording time of 8 hours. The VCE recordings were reviewed by the local VCE-responsible endoscopist and by the study coordinator $(J J K)$, to limit the possibility of missing an important lesion. The examination was considered complete if cecal visualization was achieved. To evaluate the quality of bowel preparation, a semi-quantitative evaluation by means of a grading scale (poor, moderate or good) was used. Small bowel preparation was defined as good if the mucosa was generally clean with no or minimal residual fluid and debris; moderate, if the visualization of the mucosa was impaired because of moderate fluid and debris; and poor if the mucosa visualization was highly limited because of excessive residual fluid and debris. If polyps with an estimated size of at least $1 \mathrm{~cm}$ or significant abnormalities were seen on the VCE recordings, additional endoscopic procedures were performed. Significant abnormalities were defined as: (1) polyps of any size not resembling lymfangiectasia(s) (2) ulcerative lesions or (3) abnormalities with unknown significance (like hematin). The subsequent endoscopic procedure depended on the estimated localization of the detected lesion: a gastroduodenoscopy was scheduled when lesions were identified in the duodenum; if abnormalities were seen within the first twothirds of the capsule recording time an oral/antegrade balloon-assisted enteroscopy (after fasting from midnight) was chosen and in the other cases an anal/retrograde balloon-assisted enteroscopy (after bowel preparation with PEG) was performed. The aim of these endoscopic procedures was to remove polypoid lesions, or to obtain histology if removal was not possible. Biopsies were also taken if the endoscopist had doubts with respect to the benign nature of lesions.

When no lesions were found with the balloon-assisted enteroscopy and the lesion was considered to be not definitely reached, the maximum point of introduction was marked with ink and the alternative approach was scheduled.

\section{Endpoints}

The primary endpoint of this study was incidence of neoplastic small bowel lesions, defined as the percentage of patients with a small bowel adenoma or carcinoma. The following lesion characteristics were included: morphology according to the Paris Classification [10], size, location, and histology. The secondary endpoint was the number of complications following capsule endoscopy and subsequent endoscopic procedures.

\section{Statistical analysis}

IBM SPSS 23 (SPSS, Chicago, IL) was used for all data analyses. Descriptive statistics were used to determine demographic characteristics and to analyze VCE results.

\section{Results}

Of the original cohort of 200 patients, 155 (78\%) patients consented to undergo a second VCE procedure. The reasons for exclusion are shown in $\mathbf{F i g .} \mathbf{1}$.

Two patients were excluded because of SBN detected the first VCE. These findings were described in our previous report [6]. One patient was diagnosed with a TisNOMx duodenal carcinoma and subsequently underwent a pylorus-preserving pancreaticoduodenectomy. Histological examination of the resected duodenum showed high-grade dysplasia at multiple locations. The other patient had a tubulovillous duodenal adenoma with low-grade dysplasia that was removed by endoscopic mucosal resection (EMR). Afterwards annual surveillance was 
Included patients for first VCE $\mathbf{n}=\mathbf{2 0 0}$

Excluded patients $\mathrm{n}=\mathbf{4 5}$

- Small bowel stricture $(n=1)$

- Deceased $(n=3)$

- Postoperative after surgery for SBN $(n=1)$

- Colorectal carcinoma $(n=1)$

- Unknown cause of death $(n=1)$

- SBN in first VCE $(n=2)$

- Withdrawal patient $(n=17)$

- Patient moved $(n=1)$

- Logistical reasons $(n=18)$

- Other malignancy $(n=3)$

- Urothelial cancer $(n=2)$

- Multiple myeloma $(n=1)$

Analyzed patients for second VCE $\mathrm{n}=155$

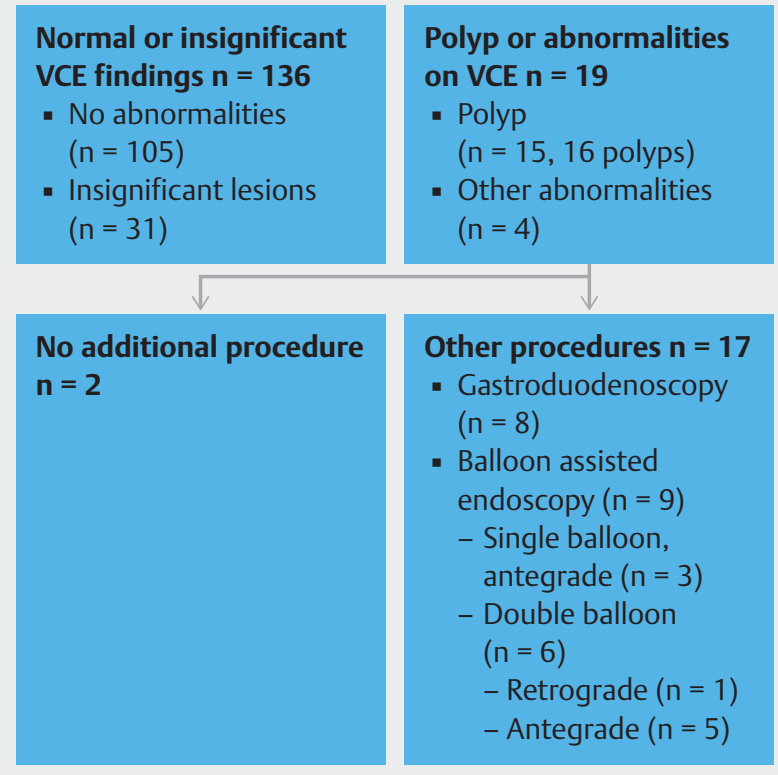

Fig. 1 Consort diagram. SBN: Small bowel neoplasia; VCE: Video capsule endoscopy

scheduled in the form of a gastroduodenoscopy with biopsy and VCE. Four years later, a flat polypoid lesion was detected at the location of the previous EMR. Endoscopic removal of the lesion was attempted, which failed due to nonlifting. A surgical resection was ultimately performed for this lesion. Histology again showed tubulovillous adenoma with low-grade dysplasia.

The included patients had a mean age of 51.6 (range 36 $71)$ years and $63(40.6 \%)$ were male. All mutations types were represented: MLH1 $(n=39)$, MSH2 $(n=57)$, Epcam $(n=2)$, MSH6 $(n=54)$, and PMS2 $(n=3)$.

The second VCE procedure was performed after a mean interval of 2.2 years (with a range of $1-6$ years) after the first procedure. A total of 22 patients underwent a follow-up procedure outside the predetermined surveillance interval. In 6 pa- tients, the second VCE procedure was performed earlier than scheduled, namely 1 to 1.5 years after the first procedure. In 16 patients, the second VCE was performed later than scheduled; in 9 patients after 3 to 4 years and in 7 patients after 4 years. Despite this deviation from the protocol, these patients were included in the analysis.

\section{Performance of VCE}

The small bowel was completely examined during VCE recording time in 134 patients ( $86 \%)$. In 3 of the patients the examination was incomplete due to technical errors. Mean gastric and small bowel transit times were $40(1-490)$ minutes and $243(41-464)$ minutes, respectively. Bowel preparation was good in the majority of patients ( $88 \%$ ); in $11 \%$ it was moderate; and in $1 \%$ it was poor. Besides possible polypoid lesions, discussed below, other findings were also detected in 31 patients: lymfangiectasia(s) ( $n=14)$, Brunner's glands $(n=3)$, angiodysplasia(s) $(n=6)$, erosion(s) $(n=8)$, intestinal metaplasia $(n=1)$, swollen mucosa $(n=1)$, a tattoo mark, $(n=1)$ and erythema $(\mathrm{n}=2)$. In 2 patients VCE also demonstrated polypoid lesions of the colon.

\section{Incidence of neoplasia}

VCE detected abnormalities in 19 patients. In 2 patients, the lesions were considered insignificant because of the small size (smaller than $1 \mathrm{~cm}$ ) and benign aspect. All significant lesions except for one were proximally located. Further endoscopic procedures were performed in 17 patients ( $\bullet$ Table 1 and - Fig. 1): 8 patients underwent gastroduodenoscopy and 9 underwent (single or double) balloon-assisted enteroscopy.

In 7 of 13 patients with possible (mostly protruded) polypoid lesions detected by VCE, no lesions were encountered endoscopically. The VCE results were considered false-positive since the location of the lesion seen on VCE was considered to be within reach of the endoscopy and no further procedures were performed. In the remaining 6 patients with polypoid lesions on VCE, endoscopic abnormalities were found. In 2 of these patients, benign-appearing lesions were found and no biopsies were taken: one had a thickened fold with a benign aspect and the other had a hypertrophic Brunner's gland. In the other 3 patients with confirmed polypoid lesions a biopsy was taken, as well as in 1 patient with swollen mucosal folds. Histologic examination showed normal mucosa $(n=2)$, lymphoid hyperplasia $(n=1)$, and a fundic gland polyp $(n=1)$. No SBN was found.

In 4 patients, endoscopic procedures were performed because of other abnormalities.

Of the 3 patients with hematin seen on VCE, 1 had nonspecific hematin confirmed by gastroduodenoscopy, another had a Helicobacter pylori-associated gastritis, and in the third no abnormalities were found. In 1 patient with a possible ulcer in the jejunum, a double balloon-assisted enteroscopy was performed, which showed no abnormalities.

\section{Complications}

No complications were observed during or following the VCE procedure or the subsequent endoscopic procedures. 
- Table 1 Results of further procedures investigating possible lesions detected by VCE.

\begin{tabular}{|c|c|c|c|c|c|}
\hline Age, sex & Mutation & VCE finding & Location & Procedure & Final diagnosis \\
\hline $64, \mathrm{~F}$ & $\mathrm{MSH} 2$ & Subpedunculated polyp $>10 \mathrm{~mm}$ & Jejunum & DBE antegrade & No abnormalities \\
\hline $59, \mathrm{M}$ & MLH1 & Sessile polyp $6-9 \mathrm{~mm}$ & Duodenum & Gastroduodenoscopy & Brunner's gland (No histology) \\
\hline $61, F$ & $\mathrm{MSH} 2$ & Subpedunculated polyp $<5 \mathrm{~mm}$ & Duodenum & Gastroduodenoscopy & $\begin{array}{l}\text { Pedunculated polyp }<5 \mathrm{~mm} \text { : } \\
\text { Normal mucosa }\end{array}$ \\
\hline $54, \mathrm{M}$ & MLH1 & Dubious polyp $<5 \mathrm{~mm}$ & Ileum & DBE retrograde & No abnormalities \\
\hline $40, F$ & MSH6 & Pedunculated polyp $>10 \mathrm{~mm}$ & Jejunum & SBE antegrade & $\begin{array}{l}\text { Pedunculated polyp 6-9 mm: } \\
\text { Lymfoid hyperplasia }\end{array}$ \\
\hline $38, F$ & MLH1 & $\begin{array}{l}\text { Two lesions } \\
\text { 1. Pedunculated polyp }<5 \mathrm{~mm} \\
\text { 2. Pedunculated polyp }<5 \mathrm{~mm}\end{array}$ & $\begin{array}{l}\text { Stomach } \\
\text { Duodenum }\end{array}$ & SBE antegrade & $\begin{array}{l}\text { Pedunculated polyp }<5 \mathrm{~mm} \text { : } \\
\text { Fundic gland polyp } \\
\text { No abnormalities }\end{array}$ \\
\hline $44, \mathrm{M}$ & MSH6 & Dubious polyp $<5 \mathrm{~mm}$ & Jejunum & DBE antegrade & $\begin{array}{l}\text { Thickened non-suspicious mucosa } \\
\text { (No histology) }\end{array}$ \\
\hline $60, \mathrm{~F}$ & PMS2 & Pedunculated polyp $<5 \mathrm{~mm}$ & Jejunum & SBE antegrade & No abnormalities \\
\hline $54, \mathrm{~F}$ & MLH1 & Flat elevation of mucosa $<5 \mathrm{~mm}$ & Duodenum & Gastroduodenoscopy & No abnormalities \\
\hline $39, F$ & MSH6 & Subpedunculated polyp 6-9mm & Duodenum & Gastroduodenoscopy & Swollen mucosa: Normal mucosa \\
\hline $55, F$ & $\mathrm{MSH} 2$ & Subpedunculated polyp 6-9mm & Jejunum & DBE antegrade & No abnormalities \\
\hline $67, \mathrm{M}$ & MSH6 & Flat elevation of mucosa $<5 \mathrm{~mm}$ & Jejunum & DBE antegrade & No abnormalities \\
\hline $44, \mathrm{M}$ & MSH6 & $\begin{array}{l}\text { Lymphangiectasia or flat elevation } \\
\text { of mucosa }<5 \mathrm{~mm}\end{array}$ & Duodenum & Gastroduodenoscopy & No abnormalities \\
\hline $57, \mathrm{M}$ & MSH6 & Hematin & Duodenum & Gastroduodenoscopy & No abnormalities \\
\hline $63, \mathrm{M}$ & PMS2 & Hematin & Stomach & Gastroduodenoscopy & Aspecific hematin \\
\hline $40, F$ & MLH1 & Ulcer & Jejunum & DBE antegrade & No abnormalities \\
\hline $49, \mathrm{M}$ & $\mathrm{MSH} 2$ & Hematin & Stomach & Gastroduodenoscopy & $\begin{array}{l}\text { Multiple small elevated lesions } \\
\text { antrum: H. pylori- associated } \\
\text { chronic focally active gastritis }\end{array}$ \\
\hline
\end{tabular}

\section{Discussion}

In the first part of this study, published earlier, we demonstrated a prevalence of small bowel neoplasia of $1.5 \%$ (3/200) in asymptomatic LS patients. Neoplastic lesions were all detected in males over 50 years of age, and all lesions were located in the duodenum. Two of the three neoplastic lesions were detected by VCE. During follow-up, it became clear that 1 T2N0M0 adenocarcinoma had been missed by VCE [6].

The aim of the present study was to determine the incidence of SBN in asymptomatic LS patients by VCE a mean of 2 years after our recent analysis of the prevalence in the same cohort. We found that after biennial surveillance none of the 155 patients had developed SBN or were diagnosed with a potentially missed lesion by the first VCE. No complications were observed due to VCE.

This is the first study to prospectively assess the incidence of SBN in LS patients. In the general population, the reported incidence of small bowel cancer is 1.4 per 100000 for men and 1.0 per 100000 for women in the United States[11]. In our study cohort, the incidence was 0 . An explanation for this difference might be that the study group was too small to determine the incidence of neoplasia. Also, the predetermined study interval of 2 years may have been too short to develop neoplasia because the SBN prevalence appeared to be lower than previously expected [6]. It is also possible that neoplastic lesions were missed by VCE. The other limitations of the study design were already discussed in detail with the baseline results [6]. One other minor limitation was the fact that no biopsies were taken in 1 patient with a thickened duodenal fold. This probably represented hypertrophic Brunner's gland tissue, but that was not confirmed by histology. The endoscopic assessment of lesions was left to the appreciation of lesions by the endoscopist.

Should surveillance of the small bowel by VCE become standard protocol for LS patients? VCE can be considered as a safe endoscopic procedure and is generally well tolerated. However, initial prevalence and 2-year incidence of SBN were low in our studies. In addition, VCE demonstrated a high number of false positive results, and we also observed 1 case of SBN that was missed by the first VCE [6]. In conclusion, our data do not support use of VCE as a surveillance technique for examining the small bowel in asymptomatic LS patients. 


\section{Acknowledgements}

This work benefited from grants from the Dutch Cancer Society (RUG 2008-4187) and Given Imaging.

Competing interests

None

\section{References}

[1] Lynch HT, Lynch PM, Lanspa S] et al. Review of the Lynch syndrome: history, molecular genetics, screening, differential diagnosis and medicolegal ramifications. Clin Genet 2009; 76: 1-18

[2] Koornstra JJ, Mourits M], Sijmons RH et al. Management of extracolonic tumours in patients with Lynch syndrome. Lancet Oncol 2009; 10: $400-408$

[3] Koornstra J], Kleibeuker JH, Vasen HF. Small-bowel cancer in Lynch syndrome: is it time for surveillance? Lancet Oncol 2008; 9: 901 - 905
[4] ten Kate GL, Kleibeuker JH, Nagengast FM et al. Is surveillance of the small bowel indicated for Lynch syndrome families? Gut 2007; 56: $1198-1201$

[5] Rodriguez-Bigas MA, Vasen HF, Lynch HT et al. Characteristics of small bowel carcinoma in hereditary nonpolyposis colorectal carcinoma. International Collaborative Group on HNPCC. Cancer 1998; 83: 240 244

[6] Haanstra JF, Al-Toma A, Dekker E et al. Prevalence of small-bowel neoplasia in Lynch syndrome assessed by video capsule endoscopy. Gut 2015; 64: 1578-1583

[7] Saurin JC, Pilleul F, Soussan EB et al. Small-bowel capsule endoscopy diagnoses early and advanced neoplasms in asymptomatic patients with Lynch syndrome. Endoscopy 2010; 42: 1057-1062

[8] Vasen HF, Abdirahman M, Brohet R et al. One to 2-year surveillance intervals reduce risk of colorectal cancer in families with Lynch syndrome. Gastroenterology 2010; 138: 2300-2306

[9] Järvinen H], Aarnio M, Mustonen $\mathrm{H}$ et al. Controlled 15-year trial on screening for colorectal cancer in families with hereditary nonpolyposis colorectal cancer. Gastroenterology 2000; 118: 829-834

[10] Endoscopic Classification Review Group. Update on the Paris classification of superficial neoplastic lesions in the digestive tract. Endoscopy 2005; 37: $570-578$

[11] Pan SY, Morrison H. Epidemiology of cancer of the small intestine. World J Gastrointest Oncol 2011; 3: 33-42 